\title{
Treatment outcome and long-term follow- up of central nervous system germ cell tumor using upfront chemotherapy with subsequent photon or proton radiation therapy: a single tertiary center experience of 127 patients
}

Kyung Taek Hong ${ }^{1,2,3+}$, Da Hye Lee ${ }^{2 \dagger}$, Bo Kyung Kim ${ }^{1,2,3}$, Hong Yul An ${ }^{1,2,3}$, Jung Yoon Choi ${ }^{1,2,3}$, Ji Hoon Phi ${ }^{4,5}$, Jung-Eun Cheon ${ }^{6}$, Hyoung Jin Kang ${ }^{1,2,3}$, Seung-Ki Kim ${ }^{4,5}$, Joo-Young Kim, Sung-Hye Park, II Han Kim ${ }^{3,9}$ and Hee Young Shin ${ }^{1,2,3,10^{*}}$ (i)

\footnotetext{
Abstract

Background: Central nervous system germ cell tumors (CNS GCTs) are a heterogeneous group of brain tumors, which are more common in Asian countries. There have been different therapeutic strategies in treating germinoma and non-germinomatous germ cell tumors (NGGCT), depending on prognosis. Moreover, long-term follow up should be emphasized due to higher late complication rates. Here, we investigated long-term outcomes and complication profiles of 127 CNS GCT patients who received uniform upfront chemotherapy.

Methods: We retrospectively evaluated outcomes of CNS GCT patients treated in Seoul National University Children's Hospital from August 2004 to April 2019. Patients were classified as low risk (LR) or high risk (HR) based on pathologic diagnosis and tumor markers. Most patients received upfront systemic chemotherapy with carboplatin, cyclophosphamide, etoposide, and/or bleomycin, followed by either proton or photon radiation therapy according to patients' choice.

(Continued on next page)
}

\footnotetext{
*Correspondence: hyshin@snu.ac.kr

${ }^{\dagger}$ Kyung Taek Hong and Da Hye Lee contributed equally to this work.

'Departments of Pediatrics, Seoul National University College of Medicine, Seoul, Republic of Korea

2Division of Pediatrics, Seoul National University Children's Hospital, Seoul, Republic of Korea

Full list of author information is available at the end of the article
}

(C) The Author(s). 2020 Open Access This article is licensed under a Creative Commons Attribution 4.0 International License, which permits use, sharing, adaptation, distribution and reproduction in any medium or format, as long as you give appropriate credit to the original author(s) and the source, provide a link to the Creative Commons licence, and indicate if changes were made. The images or other third party material in this article are included in the article's Creative Commons. licence, unless indicated otherwise in a credit line to the material. If material is not included in the article's Creative Commons licence and your intended use is not permitted by statutory regulation or exceeds the permitted use, you will need to obtain permission directly from the copyright holder. To view a copy of this licence, visit http://creativecommons.org/licenses/by/4.0/ The Creative Commons Public Domain Dedication waiver (http://creativecommons.org/publicdomain/zero/1.0/) applies to the data made available in this article, unless otherwise stated in a credit line to the data. 
(Continued from previous page)

Results: The median age at diagnosis was 11.9 (range, 3.8-25.1) years, and 54.3\% of patients were LR. Photon and proton radiation therapy were administered to 73.2 and $25.2 \%$ of patients, respectively. In both LR and HR groups, there were no significant differences in survival between photon and proton radiation therapy. The 10-year relapse incidences were 9.3 and 5.6\% in the LR and HR groups, respectively. All recurrences, except one, were local relapse. Six secondary malignancies occurred; the 10-year incidences of secondary malignancy were 2.2 and $7.6 \%$ in the LR and HR groups, respectively. The 10-year overall survival rates were $98.3 \pm 1.7$ and $91.8 \pm 3.9 \%$ in the $L R$ and $H R$ groups, respectively. In a subgroup analysis of $H R$ group, pathologically diagnosed NGGCT patients $(n=20)$ showed worse 10-year EFS $(65.9 \pm 11.9 \%, p<0.001)$ and $\operatorname{OS}(77.9 \pm 9.8 \%, p=0.024)$ rates compared to other HR patients who were not pathologically diagnosed or were confirmed as germinoma with elevated tumor markers. All mortalities were related to disease progression or secondary malignancy.

Conclusion: The strategy of treating CNS GCTs with upfront chemotherapy according to risk groups resulted in good clinical outcomes and acceptable relapse incidence. However, further modification in the definition of the HR group is needed to reduce long-term complications.

Keywords: Germ cell tumor, Central nervous system, Intracranial, Secondary malignant neoplasm, Proton therapy

\section{Background}

Childhood central nervous system germ cell tumors (CNS GCTs) are a heterogeneous group of brain tumors that are more common in Asian countries than in Western countries. In South Korea, CNS GCTs account for 16\% of all primary CNS tumors in individuals aged below 15 years, whereas in Western countries, they approximately account for $3-4 \%$ [1].

Generally, they are classified into germinoma and nongerminomatous germ cell tumors (NGGCTs). Germinoma is known to be curable with craniospinal irradiation (CSI) and local boost radiation therapy alone. However, the use of neoadjuvant or upfront chemotherapy allows for reduced radiation therapy doses and volumes, which subsequently reduces long-term radiation therapy-related adverse effects. Whole ventricle irradiation followed by a boost to the primary site is generally accepted for localized germinomas $[2,3]$, whereas CSI with local boost could be administered for metastatic germinomas [4]. Although localized germinoma shows good outcomes, chemotherapy alone or focal irradiation without whole ventricle is not an effective treatment option [5-7].

For NGGCT, various upfront chemotherapy regimens with CSI have been used. In Children's Oncology Group trial, neoadjuvant chemotherapy using carboplatin, etoposide, and ifosfamide and subsequent radiation therapy with CSI (36 Gy) and local boost (54 Gy) resulted in a 5-year event-free survival (EFS) rate of $84 \pm 4 \%$ and 5-year overall survival (OS) rate of $93 \pm 3 \%$ [8]. For nonmetastatic NGGC $\mathrm{T}$, focal irradiation was only used with neoadjuvant chemotherapy using cisplatin, etoposide, and ifosfamide in SIOPCNS-GCT-96 trial. The 5-year EFS and OS rates for localized NGGCT were $72 \pm 4$ and $82 \pm 3 \%$, respectively [9].

Given that CNS GCTs have relatively better outcomes than other pediatric brain tumors, the need to reduce long-term complications, while preserving clinical outcomes, has been emphasized. Here, we report the longterm outcomes and complication profiles of 127 patients with CNS GCTs in a single tertiary center, where uniform upfront chemotherapy had been administered.

\section{Methods}

\section{Patient characteristics}

We retrospectively reviewed the data of 127 patients diagnosed with CNS GCTs at Seoul National University Children's Hospital from August 2004 to April 2019. From August 2004, current upfront chemotherapy regimen has been used. Before receiving upfront chemotherapy, patients were classified as low risk (LR) or high risk (HR) according to pathologic diagnosis and tumor markers. LR was defined as patients with pure germinoma with normal $\alpha$-fetoprotein (AFP) and $<50 \mathrm{mIU} /$ $\mathrm{mL} \beta$-human chorionic gonadotropin ( $\beta$-HCG) levels in the serum and/or cerebrospinal fluid; the remaining patients were classified as HR. The classification of all patients and the overall treatment scheme are shown in Fig. 1. The median follow-up periods were 8.4 years (range, 0.3-14.1) in the LR group and 8.3 years (range, $0.2-14.2$ ) in the HR group, respectively.

The co-primary outcomes of this study were EFS and OS rates of each risk group. Secondary outcomes were relapse incidence, late complications, and the cumulative incidence of secondary malignant neoplasms (SMNs). The Institutional Review Board approved the procedure of reviewing medical records, and obtaining consent was waived (H-1906-155-1045).

\section{Upfront chemotherapy and radiotherapy}

The majority of patients received upfront systemic chemotherapy first. All medications were administered 


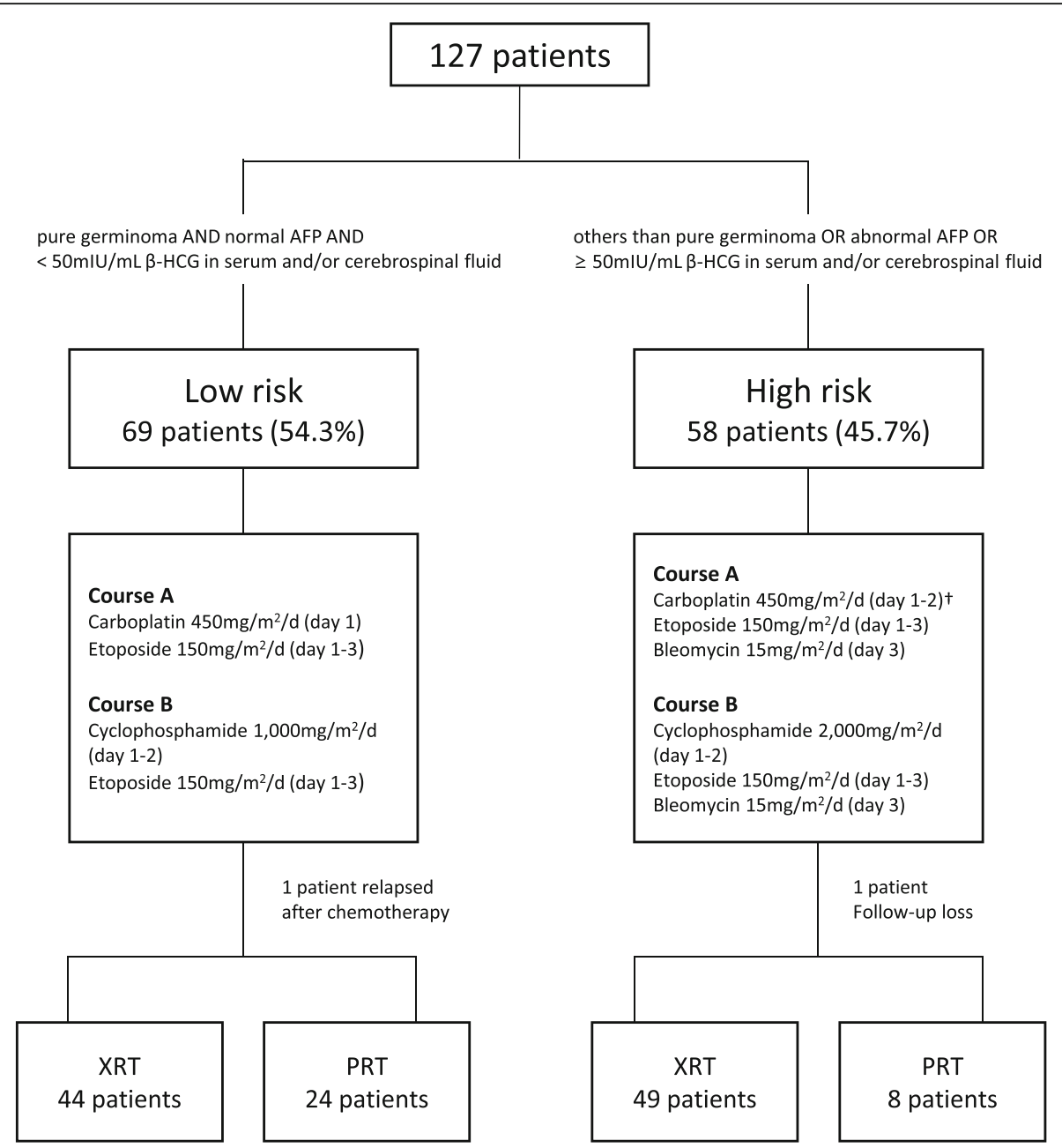

Fig. 1 Overall treatment scheme and classification of patients. Upfront chemotherapy alternating between courses A and B, and a total of 4 cycles of chemotherapy (A-B-A-B) were administered. Subsequent radiation therapy was administered using photon (XRT) or proton (PRT) therapy according to the patients' choice. TCarboplatin $450 \mathrm{mg} / \mathrm{m}^{2}$ was administered on day 1 until 2008, whereas the same dose of carboplatin was administered on days 1-2 from 2009

intravenously once daily. For the LR group, course A comprised carboplatin $450 \mathrm{mg} / \mathrm{m}^{2}$ on day 1 and etoposide $150 \mathrm{mg} / \mathrm{m}^{2}$ on days $1-3$, and course $B$ included cyclophosphamide $1000 \mathrm{mg} / \mathrm{m}^{2}$ on days $1-2$ and etoposide $150 \mathrm{mg} / \mathrm{m}^{2}$ on days $1-3$. For the HR group, course A comprised carboplatin $450 \mathrm{mg} / \mathrm{m}^{2}$ on day 1 until 2008, whereas the same dose of carboplatin was administered on days 1-2 from 2009 in order to increase the therapeutic effect, etoposide $150 \mathrm{mg} / \mathrm{m}^{2}$ was administered on days $1-3$ and bleomycin $15 \mathrm{mg} / \mathrm{m}^{2}$ on day 3 . Course B for the HR group included cyclophosphamide $2000 \mathrm{mg} / \mathrm{m}^{2}$ on days $1-2$, etoposide $150 \mathrm{mg} / \mathrm{m}^{2}$ on days $1-3$, and bleomycin $15 \mathrm{mg} / \mathrm{m}^{2}$ on day 3 . Alternating between courses A and B, a total of 4 chemotherapy treatments were performed with a 3-week interval. After the final chemotherapy cycle (second course B), subsequent photon radiation therapy (XRT; Varian, Palo Alto, CA) was conducted in our institution. If the patient wanted to receive proton radiation therapy (PRT; IBA, Louvain-La-Neuve, Belgium), it was conducted at another institution (National Cancer Center). The range of radiation therapy was described as CSI, whole-brain, whole ventricle and primary sites. CSI, whole-brain or whole ventricle irradiation were applied mutually exclusively.

\section{Statistical analysis}

Categorical and continuous variables were compared using Pearson's chi-square test or Fisher's exact test and the Student's t-test, respectively. The incidence rates of relapse and SMNs were calculated using a cumulative incidence function. Progression-free survival (PFS), EFS, and OS were analyzed using the Kaplan-Meier method. Events were defined as death, relapse, or SMNs. 
Differences in survival rates were investigated using a logrank test. $p<0.05$ was considered statistically significant. Statistical analyses were conducted using $\mathrm{R}$ version 3.2.2 (www.r-project.org) and SPSS 23.0 (IBM-SPSS, Armonk, NY).

\section{Results}

\section{Patient characteristics and tumor markers}

Among the 127 cases, 69 patients (54.3\%) were LR, and 58 patients $(45.7 \%)$ were HR. The median age at diagnosis of LR and HR groups were 11.8 (range, 3.8-18.9) and $12.2(4.5-25.1)$ years, respectively (Table 1$)$. The initial presentation and tumor location were not different between both groups. Of all patients, pathologic diagnosis was confirmed in 100 patients (78.7\%), of whom 80 patients had germinoma. The remaining 27 patients were diagnosed by the results of tumor markers and typical image findings. In LR group $(n=69), 66$ patients were germinoma and 3 were not pathologically confirmed. Because the tumor markers were normal, the latter 3 patients needed a biopsy. However the risk was high for biopsy and the symptoms progressed rapidly, thus biopsy was omitted. In HR group $(n=58), 14$ patients were germinoma and 24 were not pathologically confirmed. Of

Table 1 Characteristics of all patients $(N=127)$

\begin{tabular}{|c|c|c|c|}
\hline Characteristics & Low risk $(n=69)$ & High risk $(n=58)$ & $p$ value \\
\hline Sex & & & 0.852 \\
\hline Male & $55(79.7 \%)$ & $47(81.0 \%)$ & \\
\hline Female & $16(20.3 \%)$ & $11(19.0 \%)$ & \\
\hline Age, median (range) & $11.8(3.8-18.9)$ & $12.2(4.5-25.1)$ & 0.606 \\
\hline \multicolumn{4}{|l|}{ Initial presentation } \\
\hline Headache & $35(50.7 \%)$ & $35(60.3 \%)$ & 0.278 \\
\hline Vomiting & $28(40.6 \%)$ & $28(48.3 \%)$ & 0.384 \\
\hline Endocrine problems & $21(30.4 \%)$ & $18(31.0 \%)$ & 0.942 \\
\hline Visual problems & $19(27.9 \%)$ & $19(32.8 \%)$ & 0.557 \\
\hline Neurologic symptoms & $20(29.0 \%)$ & $14(24.1 \%)$ & 0.539 \\
\hline Tumor location & & & 0.223 \\
\hline Pineal & $24(34.8 \%)$ & $28(47.5 \%)$ & \\
\hline Sellar or suprasellar & $14(20.3 \%)$ & $13(22.0 \%)$ & \\
\hline Thalamus or basal ganglia & $17(24.6 \%)$ & $7(11.9 \%)$ & \\
\hline Ventricle & $1(1.4 \%)$ & $1(1.7 \%)$ & \\
\hline Bifocal & $10(14.5 \%)$ & $7(11.9 \%)$ & \\
\hline Multifocal & $3(4.3 \%)$ & $1(1.7 \%)$ & \\
\hline Brain stem & $0(0.0 \%)$ & $1(1.7 \%)$ & \\
\hline Pathologic diagnosis & & & $<0.001$ \\
\hline Germinoma & $66(95.7 \%)$ & $14(23.7 \%)$ & \\
\hline Immature teratoma & $0(0.0 \%)$ & $5(8.5 \%)$ & \\
\hline Choriocarcinoma & $0(0.0 \%)$ & $1(1.7 \%)$ & \\
\hline Mixed & $0(0.0 \%)$ & $14(23.7 \%)$ & \\
\hline Unknown & $3(4.3 \%)$ & $24(40.7 \%)$ & \\
\hline Tumor seeding on MRI, Yes & $7(10.1 \%)$ & $7(12.3 \%)$ & 0.704 \\
\hline Initial hydrocephalus, Yes & $39(56.5 \%)$ & $30(51.7 \%)$ & 0.589 \\
\hline ETV, Yes & $28(40.6 \%)$ & $23(39.7 \%)$ & 0.916 \\
\hline EVD, Yes & $24(34.8 \%)$ & $14(24.1 \%)$ & 0.192 \\
\hline Initial EVD and subsequent VP shunt insertion, Yes & $5(7.2 \%)$ & $6(10.3 \%)$ & 0.536 \\
\hline Direct VP shunt insertion, Yes & $5(7.2 \%)$ & $4(6.9 \%)$ & 0.939 \\
\hline Operation due to growing teratoma, Yes & $2(2.9 \%)$ & $12(20.7 \%)$ & 0.001 \\
\hline Follow-up year, median (range) & $8.4(0.3-14.1)$ & $8.3(0.2-14.2)$ & 0.706 \\
\hline
\end{tabular}

NGGCT nongerminomatous germ cell tumor, MRI Magnetic resonance imaging, ETV endoscopic third ventriculostomy, EVD extraventricular drainage, $V P$ ventriculoperitoneal 
the remaining 20 pathologic-confirmed HR patients, 14 were mixed germ cell tumors, 5 were immature teratomas, and 1 was a choriocarcinoma.

Between both groups, the frequency of initial hydrocephalus, endoscopic third ventriculostomy, and extraventricular drainage (EVD) was similar. Three patients with inserted EVD received the first cycle of chemotherapy after which EVD could be successfully removed 2 or 3 weeks after the initiation of chemotherapy without any complications or subsequent ventriculoperitoneal shunt insertion. Due to growing teratoma syndrome, 2 patients of LR group and 12 patients of HR group were operated. The pathology of 9 patients were mature teratoma, while the remaining 3 patients had immature teratoma. The median follow-up time of LR and HR patients were 8.4 and 8.3 years, respectively (Table 1 ).

Serum/cerebrospinal fluid (CSF)-AFP and $\beta$-HCG levels were examined in all available patients $(n=124)$. Only serum tumor markers were evaluated in the remaining 3 patients. The median serum and CSF-AFP levels were 1.3 (range, $0-16.1$ ) $\mathrm{ng} / \mathrm{mL}$ and 0 (range, 0 4.0) $\mathrm{ng} / \mathrm{mL}$, respectively, in the LR groups and 11.1 (range, 0-5470) ng/mL and 0.7 (range, 0-1616) $\mathrm{ng} / \mathrm{mL}$, respectively, in the HR groups. Moreover, the median serum and CSF- $\beta$-HCG levels were 0.4 (range, 0-23.0) $\mathrm{mIU} / \mathrm{mL}$ and 1.9 (range, $0-43.5) \mathrm{mIU} / \mathrm{mL}$, respectively, in the LR groups and 18.5 (range, $0-26,760$ ) $\mathrm{mIU} / \mathrm{mL}$ and 87.7 (range, $0-70,420) \mathrm{mIU} / \mathrm{mL}$, respectively, in the HR groups.

\section{Radiotherapy}

After 4 cycles of upfront chemotherapy, subsequent radiotherapy was given if the tumor did not progress. According to patients' choice, XRT at our institution or PRT at another institution (National Cancer Center) were carried out. Most of the reasons for choosing PRT were expectations that it could reduce long-term complications. The area and dose of radiation were heterogeneous due to the differences in responses to upfront chemotherapy and the presence of tumor seeding. Generally, CSI was conducted in patients with germinoma with leptomeningeal or ventricular seeding, or those diagnosed with NGGCT. However, radiotherapy could be adapted to the response to upfront chemotherapy.
Of the 68 patients in the LR group, 44 (64.7\%) and 24 (35.3\%) patients received XRT and PRT, respectively. One patient was not included because the tumor recurred after chemotherapy. Craniospinal irradiation (CSI, median dose 23.4 Gy, range 19.8-36.0), wholebrain irradiation (median dose $30.6 \mathrm{~Gy}$, range 23.4-39.6), and whole ventricle irradiation (median dose $19.8 \mathrm{~Gy}$, range 16.2-45.0) were performed in 21 (30.9\%), 2 (2.9\%), and $43(65.2 \%)$ patients, respectively. Two patients were irradiated only on the primary site. The median total dose of primary site in the LR group was 40.4 (range, 27.0-59.2) Gy. The main reasons for CSI were either seeding on MRI (magnetic resonance imaging), multifocal initial lesions, or relatively poor response to upfront chemotherapy.

Of the 57 patients in the HR group, 49 (86.0\%) and 8 (14.0\%) patients received XRT and PRT, respectively. Forty-six $(80.7 \%)$ patients received CSI (median dose 23.4 Gy, range 18.0-36.0), while 1 (1.8\%) and 10 (17.5\%) patients received whole-brain (30.6 Gy) and whole ventricle irradiation (median 23.4 Gy, range 18.0-39.6), respectively. The median total dose of primary site in the HR group was 54.0 Gy (range, 27.0-59.4), which is significantly higher than that of the LR group (40.4 Gy [range, 27.0-59.2], $p<0.001$ ). Eleven patients (19.3\%) omitted CSI, all of which had no MRI seeding and had good reactions after the initial chemotherapy or preoperative resection of the tumor. Only two of them were pathologically proven to be NGGCT. Moreover, in a subanalysis of the HR group, 14 patients who were pathologically diagnosed as germinoma with elevated tumor markers received less total dose of radiation therapy than the groups who had pathologically confirmed NGGCT or unknown histology (Table 2).

\section{Response and relapse}

After the completion of chemotherapy and radiotherapy, 92.8 and $91.2 \%$ of the LR and HR groups, respectively, showed complete or partial response by MRI. The 10year relapse incidences were 9.3 and $5.6 \%$ in the LR and HR groups, respectively. Among 5 relapsed patients in the LR group, 4 patients had germinoma of basal ganglia. Two of them received radiation therapy only on the primary site, and none of the patients who received the CSI had relapsed. The relapsed sites were all different

Table 2 Radiation field and dose of HR group $\left(N=57^{\mathrm{a}}\right)$

\begin{tabular}{|c|c|c|c|c|}
\hline & \multicolumn{3}{|c|}{ Pathologic diagnosis of HR group } & \multirow[t]{2}{*}{$p$ value } \\
\hline & Germinoma $(n=14)$ & Not confirmed $(n=23)$ & NGGCT $(n=20)$ & \\
\hline Number of patients who received CSI & $9(64.3 \%)$ & $19(82.6 \%)$ & $18(90.0 \%)$ & 0.181 \\
\hline Median dose of CSI, Gy (range) & $23.4(19.8-25.2)$ & $23.4(18.0-36.0)$ & $23.4(19.8-36.0)$ & 0.507 \\
\hline Median dose of total radiation to the primary site, Gy (range) & $45.0(27.0-54.0)$ & $54.0(30.6-59.4)$ & $54.0(39.6-54.0)$ & 0.001 \\
\hline
\end{tabular}

NGGCT nongerminomatous germ cell tumor, CSI craniospinal irradiation, Gy gray

${ }^{\mathrm{a}}$ Of total 58 patients of HR group, 57 patients were included 
from the primary site. Particularly, 1 case of relapse presented in the form of a mass in the biopsy track after the end of upfront chemotherapy. All 3 relapsed patients in the HR group showed local relapse and died of disease progression. None of the patients showed spinal seeding (Table 3).

\section{Treatment outcome}

The 10-year EFS and PFS rates were $88.6 \pm 4.5$ and $90.8 \pm 4.0 \%$ in the LR group, and $86.8 \pm 5.2$ and $92.2 \pm$ $3.8 \%$ in the HR group, respectively. The 10 -year OS rates in the LR and HR groups were $98.3 \pm 1.7$ and $91.8 \pm$ $3.9 \%$, respectively (Fig. 2A, B). In the LR group, remarkably, basal ganglia germinoma showed lower 10-year EFS rates compared to other parts of germinoma (68.9 \pm $13.1 \%$ versus $95.2 \pm 3.4 \%, p=0.012$ ).

In the HR group $(n=58)$, the pathologic diagnosis of 14 patients was germinoma, but they were classified into the HR group due to serum or CSF- $\beta$-HCG levels being above $50 \mathrm{mIU} / \mathrm{mL}(n=12)$, or abnormal AFP levels $(n=2)$. These patients showed 100\% EFS and OS rate at 10 years. Moreover, 24 patients who were not pathologically confirmed also had a $100 \%$ EFS and OS rate at 10 years. In a subgroup analysis, pathologically diagnosed NGGCT patients $(n=20)$ showed worse 10 -year EFS $(65.9 \pm 11.9 \%, p<0.001)$ and OS $(77.9 \pm 9.8 \%, p=0.024)$ rates compared to other groups who were not pathologically diagnosed or were confirmed as germinoma.

\section{Comparison between XRT and PRT}

In our cohort, both XRT and PRT were used, and patients were the ones to decide the type of radiotherapy. In the LR group, the 5-year EFS rate (XRT $91.9 \pm 4.5 \%$ $[n=44]$ versus PRT $94.1 \pm 5.7 \%[n=24], p=0.457)$ and OS rate (XRT $97.4 \pm 2.5 \%$ versus PRT $100 \%, p=0.485$ ) were similar. In the HR group, only 8 patients received
PRT while 49 patients received XRT. Although comparisons are difficult due to the lack of PRT cases, there were no significant differences in the 5-year EFS rate (XRT $91.3 \pm 4.2 \%$ versus PRT $80.0 \pm 17.9 \%, p=0.605$ ) and OS rate (XRT $93.3 \pm 3.8 \%$ versus PRT $75 \pm 21.7 \%$, $p=0.392$ ) between the types of radiotherapy.

\section{Late complications and secondary malignant neoplasms}

In our cohort, 7 patients experienced secondary neoplasms. Excluding 1 patient with a benign meningioma, 6 patients were diagnosed with SMN, 2 with thyroid carcinomas, 1 with acute lymphoblastic leukemia, 1 with acute myeloid leukemia, 1 with diffuse large B-cell lymphoma, and 1 with atypical meningioma. The 10year cumulative incidences of SMN were $2.2 \%$ in the LR group and $7.6 \%$ in the HR group (Fig. 3). However, thyroid carcinoma and meningioma occurred 10 years after the diagnosis of germ cell tumors, which means that long-term follow-up is essential for the surveillance of these types of SMN. A patient who had secondary acute myeloid leukemia died due to fungal pneumonia which was a complication from SMN treatment, while others were treated successfully.

Late complications, including endocrine, neurologic and ophthalmologic problems, are also critical issues. Until the last follow-up, 53.6 and $53.4 \%$ of LR and HR patients suffered from endocrine complications, while 18.8 and $15.5 \%$ of LR and HR patients showed neurologic complications, such as seizures or severe motor weakness. Moreover, persistent ophthalmologic complications occurred in 19.1 and $8.6 \%$ of LR and HR patients. The profile of late complications was not significantly different between the LR and HR groups.

\section{Discussion}

We retrospectively analyzed the treatment outcome of CNS GCTs, treated with homogenous upfront

Table 3 Characteristics of relapsed patients

\begin{tabular}{|c|c|c|c|c|c|c|}
\hline Risk & Number & Primary site & Previous radiotherapy & Relapsed site & Time to relapse (days) & Surviva \\
\hline \multirow[t]{5}{*}{ Low risk } & 1 & Rt. BG & LFRT 39.6Gy (XRT) & Lt. medial temporal lobe & 660 & DOD \\
\hline & 2 & Rt. BG & LFRT 39.6Gy (XRT) & Rt. frontal \& anterior temporal lobe & 1146 & Alive \\
\hline & 3 & Rt. BG & WW 19.8Gy + LFRT 19.8Gy (PRT) & Subependymal seeding & 885 & Alive \\
\hline & 4 & Lt. BG & W 19.8Gy + LFRT 19.8Gy (PRT) & Sellar area & 2654 & Alive \\
\hline & 5 & suprasellar & Only chemotherapy & Biopsy track & 102 & Alive \\
\hline \multirow[t]{3}{*}{ High risk } & 1 & $3 r d$ ventricle & $\begin{array}{l}\text { CSI 23.4Gy + LFRT 30.6Gy } \\
\text { (XRT) }\end{array}$ & Pineal gland & 449 & DOD \\
\hline & 2 & Pineal gland & $\begin{array}{l}\text { W 23.4Gy + LFRT } 30.6 \\
\text { (XRT) }\end{array}$ & Pineal gland & 201 & DOD \\
\hline & 3 & Pineal gland & $\begin{array}{l}\text { CSI 23.4Gy + LFRT 30.6Gy } \\
\text { (XRT) }\end{array}$ & Pineal gland & 365 & DOD \\
\hline
\end{tabular}

Rt. right, $B G$ basal ganglia, $L F R T$ limited-field radiation therapy, Gy gray, Lt. left, $D O D$ died of disease, $X R T$ photon radiation therapy, $W V$ whole ventricle, $P R T$ proton radiation therapy, CSI craniospinal irradiation 
A

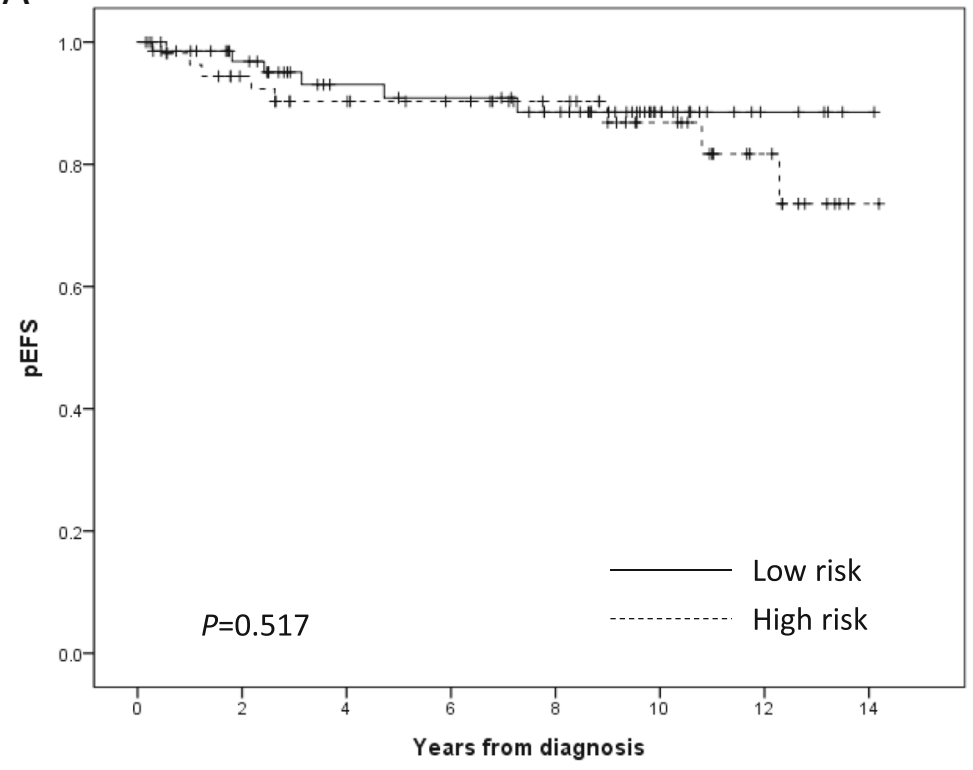

$\mathrm{B}$

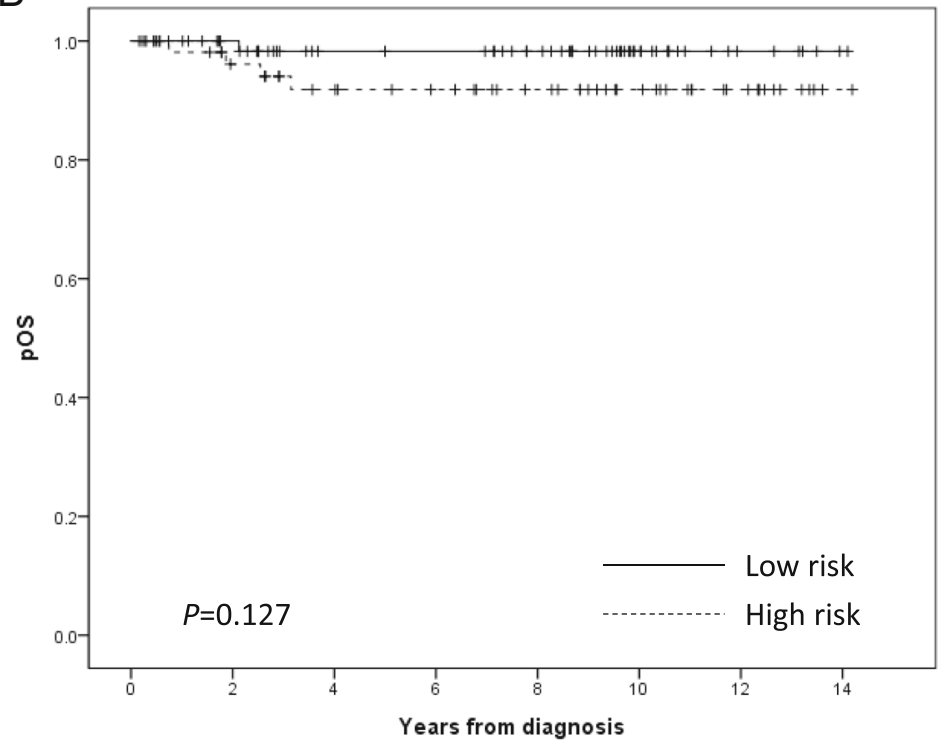

Fig. 2 The 10-year event-free survival (EFS) rates in the low risk (LR) and high risk (HR) groups were $88.6 \pm 4.5$ and $86.8 \pm 5.2 \%$, respectively (a). Events were defined as death, relapse, or secondary malignant neoplasms. The 10-year overall survival (OS) rates were $98.3 \pm 1.7$ and $91.8 \pm 3.9 \%$ in the LR and HR groups, respectively (b)

chemotherapy composed of carboplatin, cyclophosphamide, etoposide, and/or bleomycin, and radiotherapy with XRT or PRT. The dose and location of radiotherapy was not equally applied as the decision on the type of radiotherapy rested on the patients. In this study, the 10-year EFS and OS rates were 86.8-88.6 and 91.8$98.3 \%$, respectively, according to the risk group, which is comparable with the rates reported in other studies [3, $8-10]$. In particular, this study showed no significant difference in treatment outcomes between LR and HR groups.

In this study, patients were grouped as LR or HR on the basis of tumor markers and pathologic results, which is similar to the classifications used for germinoma and NGGCT in other studies $[8,9]$. There were 14 patients in the HR group who were diagnosed with pure germinoma, as determined by surgical biopsy, but showed elevated tumor markers. Their outcome was excellent, with $100 \%$ 


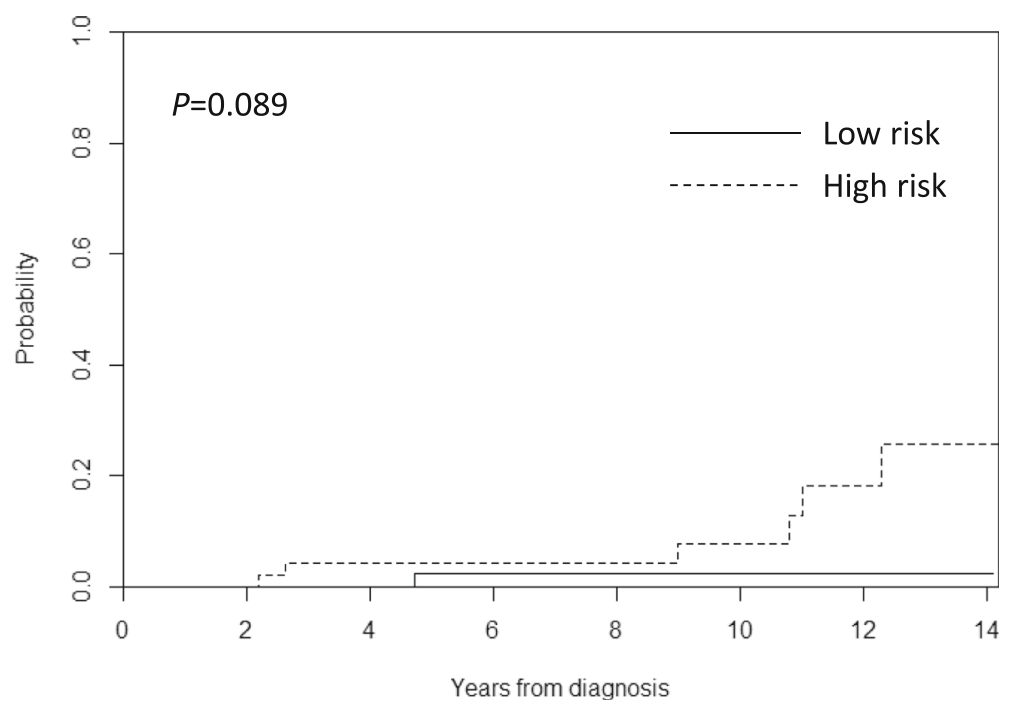

Fig. 3 The 10-year cumulative incidences of secondary malignant neoplasms were 2.2 and $7.6 \%$ in the low risk (LR) and high risk (HR) groups, respectively

of 10-year EFS and OS rates. The patients with unknown pathology also showed better treatment outcome compared to those who were diagnosed as NGGCT pathologically. Because the rise of tumor markers can mean that there are high-grade germ cell tumors other than geminoma that have not been found in biopsy, they have also received HR chemotherapy. However, our results suggest that only an increase in tumor markers does not necessarily mean a prognosis like NGGCT which has been confirmed by biopsy. Since the number of patients is too small to reach a conclusion, further research is required to elucidate whether histologically proven germinomas with elevated tumor markers could receive a de-escalated treatment. Our findings also suggest that further modification in the definition of the HR group is needed.

The majority of relapse cases of the LR group was basal ganglia germinoma. Because, half of the relapsed patients were only treated with local radiation therapy, it requires attention to interpretation of the bad prognosis of basal ganglia germinoma. Nonetheless, our finding shows the need for a sufficient radiation field in this type of tumor. In our study, heterogeneous radiation therapy was performed, and $30.9 \%$ of LR group patients were treated with CSI. There was no recurrence in all of them, but one had SMN. Therefore, considering the good prognosis of the LR group, except for basal ganglia germinoma, the application of CSI needs to be decided more carefully.

Although the number of patients was small, there were no differences in the clinical outcomes between XRT and PRT treated patients. However, given that this was a retrospective analysis, and that the type of radiotherapy was decided by the patients, it is hard to compare the 2 groups in this study. The comparison between XRT and PRT has been consistently reported in pediatric tumors [11]. One study reported no significant differences in treatment outcomes between XRT and PRT for standard risk medulloblastoma [12]. However there are reports that PRT showed better outcomes in terms of toxicity and long-term sequelae $[11,13]$. Since many factors could be involved in the selection of PRT [14], further long-term follow-up may be necessary to compare the prognosis and complications between XRT and PRT.

Among long-term complications, SMN is one of the most critical problems in childhood and adolescent cancer survivors $[15,16]$. In this study, the 10-year cumulative incidences of SMN in CNS GCTs patients were $2.2 \%$ in the LR group and $7.6 \%$ in the HR group. The most extensive cohort study of childhood cancer survivors, the Childhood Cancer Survivor Study, reported that the cumulative risk of subsequent neoplasm increased by $3.2 \%$ in 20 years and $9.3 \%$ in 30 years [17]. The mechanism by which secondary malignancy occurs is diverse. It is thought that ionizing radiation or the chemical agents used in chemotherapy increases the risk of SMN by causing DNA damage [18]. The types of cancer caused by SMN vary according to ethnicity, primary tumor distribution, and the types of chemotherapy or radiotherapy. In a multi-center retrospective study in Korean childhood cancer survivors, secondary malignancy occurred most frequently in the order of acute myeloid leukemia, myelodysplastic syndrome, thyroid cancer, and CNS tumor [19]. Among the 102 patients with SMN, 17 patients (17\%) had primary CNS tumors. In this study that is targeting only primary CNS tumors, SMN distribution was similar to that of a Korean 
retrospective study ( 2 thyroid cancers, 1 acute myeloid leukemia, 1 acute B lymphoblastic leukemia, 1 diffuse large B cell lymphoma, and 1 meningioma).

Surveillance of SMN is crucial because there may be a risk of SMNs even after successful treatment of the primary tumor. In particular, long-term surveillance strategy is essential for SMNs because the risk increases even after 20 or 30 years [20]. Indeed, the patients included in this study also developed SMNs 10 years after completing therapy. The long-term management and surveillance of SMNs should be emphasized for the improvement of overall survival. Moreover, all patients with SMN were given radiation therapy to CSA. Given the good prognosis, efforts to reduce the area and dose of radiation therapy will be necessary.

There are several limitations to our study. First, this is a retrospective study and various types and doses of radiotherapy were applied, although homogeneous upfront chemotherapy was administered in a single institution. Because PRT was not available in our institution, any patient who desired to be treated with PRT had to visit another institution. Furthermore, there were also differences in follow-up duration between patients treated with XRT and PRT. Further studies with a larger patient cohort and a longer follow-up must be performed to compare the outcome and long-term complications between XRT and PRT.

\section{Conclusion}

This study demonstrated that upfront chemotherapy using carboplatin, cyclophosphamide, etoposide and/or bleomycin according to the risk classification, along with radiotherapy, for the treatment of CNS GCTs showed good clinical outcomes and acceptable relapse incidence in our institution. However, late complications, including secondary malignancy, could substantially increase, and thus need to be thoroughly monitored. To reduce longterm complications, treatment of some HR patients, particularly those with germinoma but with high tumor markers, may be adjusted to a lower intensity.

\section{Abbreviations \\ CNS GCTs: Central nervous system germ cell tumors; LR: Low risk; HR: High risk; NGGCTs: Non-germinomatous germ cell tumors; CSI: Craniospinal irradiation; EFS: Event-free survival; OS: Overall survival; AFP: a-fetoprotein; $\beta$ - HCG: $\beta$-human chorionic gonadotropin; XRT: Photon radiation therapy; PRT: Proton radiation therapy; SMNs: Secondary malignant neoplasms; PFS: Progression-free survival; EVD: Extraventricular drainage; \\ CSF: Cerebrospinal fluid; MRI: Magnetic resonance imaging}

\section{Acknowledgments}

Not applicable.

\section{Authors' contributions}

KTH and DHL participated in study design, data collection, statistical analysis/ interpretation, manuscript drafting, and manuscript revisions. BKK, HYA, and JYC collected data. JHP, JEC, HJK, SKK, JYK, SHP, and IHK participated in manuscript editing. HJK and HYS participated in study design and manuscript editing. All authors have read and approved the manuscript.

Funding

This research was supported by a grant of the Korea Health Technology R\&D Project through the Korea Health Industry Development Institute (KHIDI),

funded by the Ministry of Health \& Welfare, Republic of Korea (grant number: HI18C0998).

\section{Availability of data and materials}

The datasets generated and analyzed during the current study are available from the corresponding author on reasonable request.

\section{Ethics approval and consent to participate}

The Institutional Review Board of the Seoul National University Hospital approved the procedure of reviewing medical records, and obtaining consent was waived because all data analyzed in this paper are anonymous (H-1906-155-1045).

\section{Consent for publication}

Not applicable.

\section{Competing interests}

The authors have no conflicts of interest to disclose.

\section{Author details}

${ }^{1}$ Departments of Pediatrics, Seoul National University College of Medicine, Seoul, Republic of Korea. ${ }^{2}$ Division of Pediatrics, Seoul National University Children's Hospital, Seoul, Republic of Korea. ${ }^{3}$ Seoul National University Cancer Research Institute, Seoul, Republic of Korea. ${ }^{4}$ Division of Pediatric Neurosurgery, Seoul National University Children's Hospital, Seoul, Republic of Korea. ${ }^{5}$ Department of Neurosurgery, Seoul National University College of Medicine, Seoul, Republic of Korea. ${ }^{6}$ Department of Radiology, Seoul National University College of Medicine, Seoul, Republic of Korea. ${ }^{7}$ Proton Therapy Center, Research Institute and Hospital, National Cancer Center, Goyang, Republic of Korea. ${ }^{8}$ Department of Pathology, Seoul National University College of Medicine, Seoul, Republic of Korea. ${ }^{9}$ Department of Radiation Oncology, Seoul National University College of Medicine, Seoul, Republic of Korea. ${ }^{10}$ Department of Pediatrics, Seoul National University College of Medicine, Seoul National University Cancer Research Institute, 101, Daehak-ro, Jongno-gu, Seoul 03080, Republic of Korea.

Received: 13 May 2020 Accepted: 2 October 2020

Published online: 09 October 2020

\section{References}

1. Ostrom QT, Gittleman H, Liao P, Vecchione-Koval T, Wolinsky Y, Kruchko C, Barnholtz-Sloan JS. CBTRUS statistical report: primary brain and other central nervous system tumors diagnosed in the United States in 2010-2014. Neuro-Oncology. 2017;19((suppl_5)):v1-v88. https://doi.org/10.1093/neuonc/ nox158.

2. Lee DS, Lim DH, Kim IH, Kim JY, Han JW, Yoo KH, Park KD, Park HJ, Chung NG, Suh CO, Kim DS. Upfront chemotherapy followed by response adaptive radiotherapy for intracranial germinoma: prospective multicenter cohort study. Radiother Oncol. 2019;138:180-6. https://doi.org/10.1016/j.radonc. 2019.06.002.

3. Alapetite C, Brisse H, Patte C, Raquin MA, Gaboriaud G, Carrie C, Habrand JL, Thiesse P, Cuilliere JC, Bernier V, Ben-Hassel M, Frappaz D, Baranzelli MC, Bouffet E. Pattern of relapse and outcome of non-metastatic germinoma patients treated with chemotherapy and limited field radiation: the SFOP experience. Neuro-Oncology. 2010;12(12):1318-25. https://doi.org/10.1093/ neuonc/noq093.

4. Calaminus G, Kortmann R, Worch J, Nicholson JC, Alapetite C, Garre ML, Patte C, Ricardi U, Saran F, Frappaz D. SIOP CNS GCT 96: final report of outcome of a prospective, multinational nonrandomized trial for children and adults with intracranial germinoma, comparing craniospinal irradiation alone with chemotherapy followed by focal primary site irradiation for patients with localized disease. Neuro-Oncology. 2013;15(6):788-96. https://doi.org/10.1093/neuonc/not019.

5. Joo JH, Park JH, Ra YS, Im HJ, Koh KN, Khang SK, Do Ahn S. Treatment outcome of radiation therapy for intracranial germinoma: adaptive radiation 
field in relation to response to chemotherapy. Anticancer Res. 2014;34(10): 5715-21.

6. Eom KY, Kim IH, Park Cl, Kim HJ, Kim JH, Kim K, Kim SK, Wang KC, Cho BG, Jung HW, Heo DS, Kang HJ, Shin HY, Ahn HS. Upfront chemotherapy and involved-field radiotherapy results in more relapses than extended radiotherapy for intracranial germinomas: modification in radiotherapy volume might be needed. Int J Radiat Oncol Biol Phys. 2008;71(3):667-71. https://doi.org/10.1016/j.jprobp.2008.01.061.

7. Balmaceda C, Heller G, Rosenblum M, Diez B, Villablanca JG, Kellie S, Maher P, Vlamis V, Walker RW, Leibel S, Finlay JL. Chemotherapy without irradiation--a novel approach for newly diagnosed CNS germ cell tumors: results of an international cooperative trial. The first international central nervous system germ cell tumor study. J Clin Oncol. 1996;14(11):2908-15. https://doi.org/10.1200/jco.1996.14.11.2908.

8. Goldman S, Bouffet E, Fisher PG, Allen JC, Robertson PL, Chuba PJ, Donahue B, Kretschmar CS, Zhou T, Buxton AB, Pollack IF. Phase II trial assessing the ability of Neoadjuvant chemotherapy with or without second-look surgery to eliminate measurable disease for Nongerminomatous germ cell tumors: a Children's Oncology Group Study. J Clin Oncol. 2015;33(22):2464-71. https://doi.org/10.1200/JCO.2014.59.5132.

9. Calaminus G, Frappaz D, Kortmann RD, Krefeld B, Saran F, Pietsch T, Vasiljevic A, Garre ML, Ricardi U, Mann JR, Gobel U, Alapetite C, Murray MJ, Nicholson JC. Outcome of patients with intracranial non-germinomatous germ cell tumors-lessons from the SIOP-CNS-GCT-96 trial. Neuro-Oncology. 2017;19(12):1661-72. https://doi.org/10.1093/neuonc/nox122.

10. Lian X, Hou X, Yan J, Sun S, Miao Z, Liu Z, Wang W, Shen J, Shen J, Hu K, Zhang F. Treatment outcomes of intracranial germinoma: a retrospective analysis of 170 patients from a single institution. J Cancer Res Clin Oncol. 2019;145(3):709-15. https://doi.org/10.1007/s00432-018-2743-0.

11. Huynh M, Marcu LG, Giles E, Short M, Matthews D, Bezak E. Current status of proton therapy outcome for paediatric cancers of the central nervous system - analysis of the published literature. Cancer Treat Rev. 2018;70:27288. https://doi.org/10.1016/j.ctrv.2018.10.003.

12. Eaton BR, Esiashvili N, Kim S, Weyman EA, Thornton LT, Mazewski C, MacDonald T, Ebb D, MacDonald SM, Tarbell NJ, Yock TI. Clinical outcomes among children with standard-risk medulloblastoma treated with proton and photon radiation therapy: a comparison of disease control and overall survival. Int J Radiat Oncol Biol Phys. 2016;94(1):133-8. https://doi.org/10. 1016/j.jijobp.2015.09.014

13. Kahalley LS, Peterson R, Ris MD, Janzen L, Okcu MF, Grosshans DR, Ramaswamy V, Paulino AC, Hodgson D, Mahajan A, Tsang DS, Laperriere N, Whitehead WE, Dauser RC, Taylor MD, Conklin HM, Chintagumpala M, Bouffet E, Mabbott D. Superior intellectual outcomes after proton radiotherapy compared with photon radiotherapy for pediatric medulloblastoma. J Clin Oncol. 2019. https://doi.org/10.1200/jco.19.01706.

14. Shen CJ, Hu C, Ladra MM, Narang AK, Pollack CE, Terezakis SA. Socioeconomic factors affect the selection of proton radiation therapy for children. Cancer. 2017;123(20):4048-56. https://doi.org/10.1002/cncr.30849.

15. Armstrong GT, Liu W, Leisenring W, Yasui Y, Hammond S, Bhatia S, Neglia JP, Stovall M, Srivastava D, Robison LL. Occurrence of multiple subsequent neoplasms in long-term survivors of childhood cancer: a report from the childhood cancer survivor study. J Clin Oncol. 2011;29(22):3056-64. https://doi.org/10.1200/JCO.2011.34.6585.

16. Doi K, Mieno MN, Shimada Y, Yoshinaga S. Risk of second malignant neoplasms among childhood cancer survivors treated with radiotherapy: meta-analysis of nine epidemiological studies. Paediatr Perinat Epidemiol. 2009;23(4):370-9. https://doi.org/10.1111/j.1365-3016.2009.01047.x.

17. Meadows AT, Friedman DL, Neglia JP, Mertens AC, Donaldson SS, Stovall M, Hammond S, Yasui Y, Inskip PD. Second neoplasms in survivors of childhood cancer: findings from the childhood cancer survivor study cohort. J Clin Oncol. 2009;27(14):2356-62. https://doi.org/10.1200/JCO.2008.21.1920.

18. Ciccia A, Elledge SJ. The DNA damage response: making it safe to play with knives. Mol Cell. 2010;40(2):179-204. https://doi.org/10.1016/j.molcel.2010.09.019.

19. Koh KN, Yoo KH, Im HJ, Sung KW, Koo HH, Kim HS, Han JW, Yoon JH, Park HJ, Park BK, Baek HJ, Kook H, Lee JA, Lee JM, Lee KC, Kim SK, Park M, Lee YH, Lyu CJ, Seo JJ. Characteristics and outcomes of second malignant neoplasms after childhood cancer treatment: multi-center retrospective survey. J Korean Med Sci. 2016;31(8):1254-61. https://doi.org/10.3346/jkms. 2016.31.8.1254.
20. Robison LL, Hudson MM. Survivors of childhood and adolescent cancer: life-long risks and responsibilities. Nat Rev Cancer. 2014;14(1):61-70. https://doi.org/10.1038/nrc3634.

\section{Publisher's Note}

Springer Nature remains neutral with regard to jurisdictional claims in published maps and institutional affiliations.
Ready to submit your research? Choose BMC and benefit from:

- fast, convenient online submission

- thorough peer review by experienced researchers in your field

- rapid publication on acceptance

- support for research data, including large and complex data types

- gold Open Access which fosters wider collaboration and increased citations

- maximum visibility for your research: over $100 \mathrm{M}$ website views per year

At BMC, research is always in progress.

Learn more biomedcentral.com/submissions 